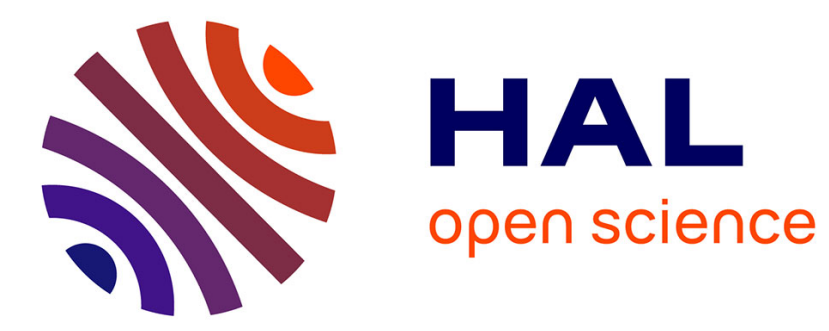

\title{
Les avatars de la publicité télévisée ou les vies rêvées des femmes
}

Jean-Claude Soulages

\section{To cite this version:}

Jean-Claude Soulages. Les avatars de la publicité télévisée ou les vies rêvées des femmes. Le Temps des médias. Revue d'histoire, 2009, 10.3917/tdm.012.0114 . hal-02614717

\section{HAL Id: hal-02614717 \\ https://hal.science/hal-02614717}

Submitted on 21 May 2020

HAL is a multi-disciplinary open access archive for the deposit and dissemination of scientific research documents, whether they are published or not. The documents may come from teaching and research institutions in France or abroad, or from public or private research centers.
L'archive ouverte pluridisciplinaire HAL, est destinée au dépôt et à la diffusion de documents scientifiques de niveau recherche, publiés ou non, émanant des établissements d'enseignement et de recherche français ou étrangers, des laboratoires publics ou privés. 


\section{Les avatars de la publicité télévisée ou les vies rêvées des femmes ${ }^{1}$}

Jean-Claude SOULAGES PR à l'ICOM Université Lumière Lyon 2

Centre Max Weber UMR 5283

Jouant sur deux scènes à la fois, la publicité est située au croisement de deux champs incommensurables, celui du marché des biens de consommation et celui de la circulation de représentations. Cette hybridité qu'elle assume sans complexe explique pour une grande part son dynamisme et sa capacité permanente au renouvellement. À tel point que, tant du point de vue des flux financiers dont elle irrigue les médias, que du point de vue des représentations imaginaires dont elle assure la circulation, elle constitue depuis le siècle dernier le décor quotidien et envahissant de la sphère publique de nos sociétés. Scène publique qui est devenue du même coup un espace de réverbération pour des croyances concernant les rôles, les identités et les pratiques sociales. Parmi celles-ci, la figure de la femme occupe une place stratégique.

Or, contrairement à ce qu'elle prétend être ou à l'image qu'on lui prête, la publicité est loin de correspondre à cette instance créatrice et originale qui introduirait des innovations ou des ruptures décisives dans nos styles de vie ou de pensée. Elle opère plutôt comme une caisse de résonance pour des discours déjà circulants dont elle s'attache à proposer un "précipité" imagé. N'oublions pas que, pour le publicitaire, il s'agit prioritairement d'offrir des points d'accroche ou bien des prises à ses destinataires d'où cet opportunisme frénétique à recycler (à récupérer) les matériaux les plus hétéroclites. Quel qu'en soit le prix du reste, car cette hybridité, évoquée plus haut, concerne en tout premier lieu son public : consommateur de marchandise en tant qu'agent économique, et dans le même temps, usager d'un média plus ou moins indifférent à la performance publicitaire. Prisonnière jusqu'à nos jours de cette ambivalence, la publicité télévisée, depuis plus d'un demi-siècle, décline aux yeux du grand public un répertoire inépuisable de modes de vie et d'attitudes auxquels elle apporte ses propres définitions. Cette vitrine de la société de consommation représente, à ce titre, pour le chercheur ${ }^{2}$ une archive prolixe sur les métamorphoses de l'individu moderne.

\section{Chacun à sa place}

L'installation et l'acceptation sociales de la télévision se sont accompagnées tout particulièrement en Europe de l'encadrement voire de l'écrasement du flux télévisuel par le poids de l'Etat-nation. En France, les pouvoirs politiques ont introduit la publicité à dose homéopathique comme une ressource financière indispensable à l'élargissement d'un réseau télévisuel étriqué, obérant du même coup à l'époque la naissance éventuelle de télévisions commerciales indépendantes. Il faut toutefois porter au crédit de cette intrusion le fait que, grâce à sa couverture globale de l'hexagone, la publicité télévisée a

\footnotetext{
${ }^{1}$ Article publié dans le Le temps des médias. La cause des femmes, MEADEL Cécile dir., Paris, éditions nouveau monde, $2009, \mathrm{n}^{\circ} 12$.

${ }^{2}$ L'Inathèque de France achève la numérisation et l'indexation de l'ensemble des spots publicitaires diffusés par les chaînes françaises qui sont désormais accessibles aux chercheurs.
} 
participé grandement à la familiarisation et à l'apprivoisement de la publicité commerciale par la majorité des Français. La marchandise et puis plus tardivement, les marques vont s'y donner en spectacle. Le consommateur à son tour va petit à petit y imposer sa présence, avec ses univers et ses tranches de vie euphoriques, mais aussi avec son côté opaque, assujetti de façon souterraine à un jeu de normes et de régulations.

Dans ses balbutiements, l'ancêtre de la publicité télévisée a assuré longtemps un rôle de simple porte-voix, relais docile de groupements d'annonceurs (instances politiques ou bien des lobbys économiques, commerciaux) qui accordaient bien peu de marge à l'activité du publicitaire. Cette publicité "primitive" ou publicité compensée ${ }^{3}$ introduite dès 1951 campe un territoire de préconisations secrétant un décor et une charte civique immuables; la santé, le bien-être, le progrès. Ses conseils sont assénés sur un ton impersonnel à des consommateurs sujets dociles, supposés réceptifs à l'intérêt et au bien commun. Les produits ou les services sélectionnés entonnent tous le même hymne à la modernité et au bien-être (l'électricité, les emprunts d'état, l'assurance-vie, la chicorée, le lait, la banane, etc.). Dans la plupart de ces scénettes minimalistes, le destinataire endosse les traits du peuple anonyme et indifférencié au cœur duquel la figure de la femme est à peine esquissée si ce n'est pour incarner la pièce rapportée d'un puzzle à mettre en ordre : les desseins ou le futur radieux de la France. Cette première époque de la réclame télévisée ne déroge pas au règne sans partage de l'ordre moral patriarcal où chacun reste à sa place: l'homme au travail, la femme à la maison. Aucune échappatoire individuelle n'est possible puisqu'il n'existe pas d'espace pour le destin personnel du consommateur. Dans ces temps archaïques, le consommateur ou la consommatrice n'ont pas encore de visage.

La métamorphose brutale de ce paysage monotone sera consécutive à l'introduction de la publicité de marques ${ }^{4}$ qui, le $1^{\text {er }}$ octobre 1968 permettra à trois premiers secteurs économiques d'accéder au petit écran : le textile, l'alimentaire et l'électroménager. Les nouveaux venus doivent impérativement se construire un univers de discours singulier et donc camper un paysage social habité par des êtres aux identités saillantes. La publicité télévisée va alors s'évertuer à aller à la rencontre de ses consommateurs et consommatrices postulés qui vont du même coup s'immiscer progressivement dans ses récits.

Cette première époque de la publicité télévisée est marquée par deux traits caractéristiques.

Le premier, c'est l'hégémonie du produit. Au cœur de cette vision épiphanique de la marchandise, la femme est omniprésente. L'appareil électroménager représente l'auxiliaire merveilleux de la quête de la "ménagère parfaite". Ce rejeton de la modernité va y devenir la véritable vedette d'un spectacle quotidien. Dans les premiers spots, la machine est installée sous les feux de la rampe au centre du studio présentée

\footnotetext{
${ }^{3}$ Les premières publicités télévisuelles datent de 1951 et sont à l'initiative d'Antoine Pinay, maire de Saint-Chamond, qui cherchait à faire la promotion du cuir français dont sa ville était dépendante. Les « émissions compensées » relevaient d'un but «d'intérêt national» et proposées par des organismes publics, semi-publics ou par des regroupements de professionnels.

${ }^{4}$ La diffusion des premières publicités de marques date du $1^{\text {er }}$ octobre 1968 sur la Une, elle intervient sur la $2^{\mathrm{e}}$ chaîne à partir de 1970 et sur la troisième chaîne en 1975 . Les eaux minérales, les jus de fruits, les appareils photos apparaissent en 1970, la publicité automobile n'est autorisée qu'en 1971.
} 
soit en voix-off par la voix mâle d'un bonimenteur, soit livrée aux bons soins et à l'enthousiasme débordant d'un personnage féminin qui ouvre l'appareil, le manipule, le caresse et sourit. Le mot d'ordre récurrent de tous les annonceurs : rendre la vie des femmes, facile ; «Il facilite la vie ! » (Brandt, 1968). L'acquisition et la possession de l'appareil impliquent un accès immédiat au bien être pour tous ; "Qui dit Radiola dit confort ménager! » (1968). «Tournez un bouton et vous êtes libre » (machine à laver Peugeot 1968) et le bouton une fois enclenché, la femme peut rejoindre son mari confortablement installé devant la télévision. Dans tous ces sketches rudimentaires, ce qui n'est pas dit (tout en s'énonçant) c'est que la vie des femmes est difficile. Or, il ne s'agit en aucun cas de la changer, mais de contribuer à la rendre plus facile. Il n'est nullement à l'ordre du jour de remettre en cause la condition féminine, la question ne se pose même pas. Le publicitaire déplace le propos et lui substitue son propre argumentaire ; la femme est un être démuni, hanté par le manque qui va rejoindre les troupes du progrès et du bien-être grâce à la magie de l'objet.

La deuxième caractéristique dominante de ces scénarios figuratifs et qui va perdurer jusqu'à la fin des années 1970 a trait au statut de la femme, toujours explicitement et la plupart du temps "accompagnée". Cet enchaînement de la consommatrice à l'univers de la marchandise et simultanément à une parcelle de la vie sociale, s'opère par la déclinaison de récits de vie illustrant à chaque fois la proposition d'un monde possible. La femme au cours de ses multiples apparitions va s'incarner sous les traits de trois figures principales rattachées à trois de ces mondes possibles; le groupe (les amies, les voisines), le couple (avec son conjoint), la famille (avec ses enfants, sa mère ou sa belle-mère).

Le premier de ces mondes possibles inscrit le personnage féminin, dans un réseau relationnel ou social de proximité exclusivement féminin. Ses semblables, ses voisines font comme elle : «Dans mon immeuble, tout le monde en prend!» (1968 les pâtes Rivoire et Carré $^{5}$ ). Ou bien ses amies la conseillent : la copine qui, face à l'accablement de son amie, la supplie « prends donc Ajax ammoniaqué !» (1976). Ou bien encore lors de la rencontre avec un bonimenteur lessivier improbable qui réussit l'exploit de mettre à contribution «toutes les femmes du village pour utiliser la nouvelle lessive XTRA » (1978). Ces prescripteurs du produit qui incarnent à chaque fois une instance sociale, tout en cautionnant le progrès et la modernité, ratifient et entérinent surtout implicitement le statut de la femme assujettie toujours à la même place dans la division du travail domestique.

Le second de ces univers correspond au couple et s'étend le plus souvent à l'espace du foyer familial. Ce dernier se caractérise par l'activisme du personnage féminin qui arpente son territoire ou bien dont la présence est attestée, métonymiquement, par des mains expertes qui accomplissent des prouesses ménagères. La plupart des publicités pour les produits alimentaires figurent une trajectoire rituelle et obligée de la femme ;

\footnotetext{
${ }^{5}$ Une voix féminine en off. À l'image, des barres d'immeubles modernes, la nuit, fenêtres allumées. La caméra se déplace d'un étage à l'autre : «Écoutez moi ! J'ai pas été voir chez les autres mais, dans mon immeuble, tout le monde en prend, ça fait d'ailleurs déjà longtemps que j'ai dit à Mme Martin de les essayer, elle les a fait goûter à Mme Langlois, une révélation! Elle est très bien avec sa voisine d'en dessous, Mme Lancier, de fil en aiguille Mme Dumons mère, je vous dirais, Madame Dumons, Mme Leroux, c'est du pareil au même, une qui en raffole, c'est la voisine du $5^{\text {ème }},(\ldots) »$. Les barres d'immeubles se transforment à la fin en un empilement de paquets de pâtes Rivoire et Carré.
} 
préparation parfaite et réussie, départ de la cuisine, arrivée à la salle à manger où les hommes et les enfants attendent impatiemment (et accessoirement applaudissent). Zèle suprême, lorsque par hasard la maîtresse de maison s'est absentée, cette dernière va consciencieusement transmettre ses consignes culinaires à son époux au téléphone (Babybell, 1968). Et miraculeusement, lorsque celui-ci est introuvable, il demeure fantomatiquement présent dans l'interpellation récurrente "Madame, votre mari pense que... », rituel obséquieux de contact, mais désignation qui induit, dans le même temps, une assignation de place, « femme de, épouse de ».

Le dernier de ces mondes possibles est circonscrit au cercle familial et correspond à l'image de la femme-mère, redoublée souvent par celle de la femme-fille car la protagoniste féminine y est également représentée sous l'identité d'une fille de ou d'une belle fille de. Dans ces récits édifiants, ces mères ou ces grand-mères aux apparitions épisodiques, si elles assurent bel et bien un pont entre la tradition et la modernité ( «la grand mère électrique » Tournus, 1978) en prodiguant leurs conseils et leurs ressources, incarnent avant tout la garantie de la perpétuation du modèle familial. Ces marâtres, souvent dépeintes en arrière-plan et secondarisées par une série de procédés de dérision, représentent les figures tutélaires de la reproduction et de la tradition. Cet avatar de la femme marâtre, gardienne des valeurs de la famille et de la transmission, va accompagner de longues années durant, sous les traits de la Mère Denis à partir de 1971 , le destin de la marque Vedette.

Ce qui domine durant cette époque "primitive" de la publicité télévisée, c'est manifestement un enfermement de l'horizon de la consommation dans une vision structurellement fonctionnelle du produit et donc des usages et du bien-être attendus qui en découleraient. Le consommateur, la consommatrice se retrouvent prisonniers d'un univers exclusif de besoins tout à fait prescriptif. Pour le personnage féminin, le monde social s'impose comme un lieu coercitif et déjà programmé pour une assignation de place, un espace clos à l'intérieur duquel il ne lui reste plus qu'à tenir un rôle. Au niveau de l'énoncé, le message s'inscrit strictement à l'intérieur du territoire du produit tandis que s'y profile la présence envahissante (et la pression supposée) de l'annonceur. Le publicitaire reste en retrait dans les coulisses. En dépit de cet effacement relatif, ce dernier contribue toutefois à la mise en lisibilité d'un fragment de l'expérience sociale et intersubjective, non pas en cherchant à la restituer dans une quelconque vérité documentarisante - cette « honnête » reflet revendiqué par beaucoup de professionnels - mais en la reconfigurant à travers des scénarios figuratifs plus ou moins réalistes en relation avec une série d'imaginaires. Cette première période du discours publicitaire porté par l'élan des « 30 glorieuses » a manifestement servi à l'apprivoisement de la vie moderne avec son décor, ses postulats, ses objets fétiches et ses désirs insatiables. Le poids de cet imaginaire de la modernité a laissé en déshérence la question des identités. Malgré la proximité de 68, la publicité télévisée se contente de transférer dans le monde merveilleux de ces objets nouveaux des modèles idéologiques du genre surannés mais encore dominants.

Du point de vue diachronique, il est toujours risqué d'avancer l'hypothèse de ruptures catégoriques dans la discursivité sociale, ici, entre ce qu'il conviendrait de désigner comme les formes d'une paléo-publicité, en empruntant les termes à Umberto 
$E{ }^{6}$, qui s'effacerait face à une néo-publicité qui surgirait à la fin des années 70 . Il apparaît plus pragmatique de relever plutôt l'existence de régimes dominants à une époque donnée, mais qui cohabitent avec d'autres modèles divergents ou minoritaires qui survivent localement liés à des types de produits (la lessive par exemple), des univers de référence (la voiture) ou bien des stratégies particulières à une marque (provocation, dérision, etc.). Les transformations que va connaître la publicité télévisée à la fin des années 70 tiennent tout autant à la maturité des professionnels qui se tournent enfin vers l'expérience vécue des publics, qu'aux métamorphoses institutionnelles qu'a connues le média télévisuel qui ont permis d'accroître considérablement l'offre de programmes et donc la diversité des messages eux-mêmes. Interviennent également des facteurs d'ordre sociologique plus décisifs sans doute liés aux glissements des représentations sociales à l'intérieur de la société française ou bien d'autres encore de nature purement esthétique ou rhétorique dépendants des innovations technologiques et formelles du média. Chacun de ces facteurs concourrant à la dynamique de ce changement.

\section{L'identité fragmentée ou les avatars publicitaires du féminin}

Cette publicité de marques plus mature, qui va petit à petit s'épanouir et s'imposer dès la fin des années 1970 sur le petit écran confère une trajectoire non plus seulement au produit mais au consommateur ou à la consommatrice. En effet, ces derniers vont partager désormais avec la marque un univers commun. Dans les années 2000 le slogan de Loréal «parce que je le vaux bien ! » représente le parangon de cette stratégie. On invente le consommateur et le destinataire du message publicitaire en même temps que le produit. Quitte à ce que le discours publicitaire opère une reconfiguration le plus souvent enchantée de l'expérience sociale et individuelle. Il fonctionne dès lors comme l'alambic d'une alchimie sémantique qui, tout en cherchant à donner un sens à l'univers trivial de la marchandise et de la consommation, s'efforce d'activer et de cristalliser dans le même temps certains affects qui relient le consommateur à son environnement représentationnel. La publicité télévisée assure alors un véritable travail de (télé)portage, de mise au jour, de traduction et de schématisation d'un fragment de la vie sociale et personnelle.

Pragmatiquement, le discours publicitaire a su au cours de sa brève histoire sur le petit écran, s'adapter à l'évolution des valeurs sociétales et tout particulièrement à celles touchant au rôle et à la visibilité sociale de la femme qui ont considérablement évolué dès la fin des années 70. Économiquement émancipée et affranchie, cette dernière se situe au cœur de la consommation pour laquelle elle assume désormais le rôle de prescriptrice à part entière. Au scénario édifiant et univoque du parcours imposé, se substitue désormais la déambulation "librement assumée", dans les avenues du bonheur et de l'épanouissement, d'une créature féminine non plus sujette mais actrice et désirante. Cette métamorphose repose sur un changement complet de point de vue. De cette fascination des grandes découvertes de la modernité, on est passé au défilé des tranches de vie et des expériences égotistes. En quelques décennies, on a basculé d'un encadrement prescriptif et unidimensionnel à une autonomie revendiquée voire à une

\footnotetext{
${ }^{6}$ Eco Umberto (1985), “De la paléo-télévision à la néo-télévision”, La guerre du faux, Paris, Grasset.
} 
déliaison apparente entre l'individu et les instances institutionnelles et patriarcales. Simultanément, s'est esquissé un processus de déconstruction et d'émiettement du personnage féminin qui n'est plus seulement la femme de (l'épouse de), l'amie de ou la mère de, la fille de. Rapidement, celui-ci va trouver à s'incarner dans de nouvelles séries d'avatars médiatiques marqués cette fois-ci par la figure récurrente et plurielle de la femme seule (non accompagnée) qui a jeté les amarres du foyer domestique et de l'autorité du mâle ou de l'escorte familiale, un être déterritorialisé, doté de sa propre trajectoire, sociale, voire amoureuse. Comme si dans l'entrelacs de ces discours s'était immiscé le simulacre d'une libération voire même l'esquisse d'une forme de parité. Or cette parité n'existe qu'en surface.

Deux raisons à cela; la première d'ordre esthético-ontologique. La production publicitaire a su plus que tout autre secteur de l'audiovisuel tirer parti de l'esthétique et des rhétoriques télévisuelles ${ }^{7}$. Certains chercheurs vont jusqu'à affirmer qu'elle a profondément marqué la télévision de son style ${ }^{8}$, clinquant et simpliste. Or, cette centration sur l'esthétisation et la simplification a un prix. En diffusant sans cesse cette bande-annonce pour une vie rêvée, la publicité tend dans une certaine mesure à reconstruire sa propre bulle d'expérience en grande partie déconnectée du social donnant naissance à un univers du «vrai-faux ${ }^{9}$ » reposant sur toute une chaîne de schématisations. Avec pour principale conséquence que, chemin faisant, dans la plupart de ses récits, le tissu social s'est dissous avec ses scènes naturalistes de bistrots, ses rencontres de rues, de paliers, etc. qu'exhibaient à profusion les publicités des années 60 et 70 . Et, s'il est de temps en temps esquissé, le paysage social dépeint dans la publicité contemporaine est de plus en plus rarement ce décor de proximité sociale spontané et populaire (peuplé d'êtres humains et de classes sociales), mais un paysage recomposé fait d'espaces de travail édulcorés, d'espaces domestiques aseptisés et de lieux publics épurés. Cette irréalisation atteint également le personnage féminin lui-même qui très souvent dans ses apparitions n'est plus situé dans un espace social marqué, mais systématiquement décontextualisé, désocialisé, déréalisé au sens propre. Cette figure individuelle et abstraite opère alors comme un masque pour une identité sociale effective comme si la publicité, non contente de vendre du rêve, opérait dans le même temps un travail d'enfouissement du réel.

La deuxième raison repose sur la fragmentation quasi schizophrénique de cette nouvelle identité féminine. Dans le dérouler de ces récits, trois figures dominantes se détachent, totalement disjointes ; l'héritière, la combattante, l'icône.

Le premier avatar de cette figuration reconduit le stéréotype patriarcal qui a marqué les débuts de la publicité télévisée. Cette «ménagère de moins de 50 ans » ciblée depuis la fin des années 80 par les diktats du marketing télévisuel ${ }^{10}$ en est la digne héritière. Ce

\footnotetext{
${ }^{7}$ Soulages Jean-Claude, Les rhétoriques télévisuelles ou le formatage du regard. Media Recherches, Paris, Bruxelles, INA De Boeck Université, 2007.

${ }^{8}$ Caldwell, John Thorton, Televisuality, style, crisis and authority in american television, Rutgers University Press, New Brunswick, New Jersey, 1995.

9 Houdebine, Anne-Marie, «Du langage de la publicité, transgressions linguistiques et représentations sociales. », Travaux de linguistique $\mathrm{n}^{\circ}$ 5-6, mai 1994, Université d'Angers, p. 57-72.

${ }^{10}$ Preuve, s'il en est de l'emprise de la publicité sur le média télévisuel, puisque c'est bien elle qui a pris les rênes de la première chaîne française privatisée. Non seulement ses récits ont l'ambition d'y capter le
} 
personnage est la figure imposée qui régit l'espace domestique et familial, à la fois femme, mère et fille. Elle vaque, le plus souvent muette, à ses occupations de "femme d'intérieur" ; la lessive, le ménage, le repas, les enfants, etc. Elle règne sans partage sur son territoire. Territoire qui se confond avec un univers de compétence exclusif au cœur duquel la parité n'existe pas. Car, a contrario, son alter ego masculin s'y montre totalement incompétent. Son irruption dans ce pré carré est toujours le fruit de concours de circonstances fortuits (sa femme, sa mère sont absentes, etc.) et ses activités y relèvent le plus souvent de l'exploit ou du défi (changer les couches, faire une lessive, etc.).

Le second avatar est celui de la combattante. Cette pionnière se déplace désormais sur des territoires de produit et des univers de compétences qui ne sont pas les "siens" comme le travail, la voiture, la séduction (la drague), etc.

Dans ces territoires excentrés par rapport à ses compétences attendues, le personnage féminin a, tout comme dans la vie réelle, à justifier sa place par des stratégies constantes d'hypercorrection, de zèle, d'écart différentiel (par rapport à son homologue masculin). Les publicitaires vont résoudre cette ambivalence en sursignifiant la présence de la femme dans ces univers où sa compétence serait constamment mise en jeu (« enfin elle y est, tout en n'y étant pas tout à fait !»). À cette fin, ces derniers utilisent deux procédés; l'humour (elle l'a fait, mais ce n'est pas sérieux!) et le récit rocambolesque («tout cela, pour ça!»). Et lorsque certains professionnels s'acharnent parfois, laborieusement à défiger les stéréotypes machistes, leur insistance (pour la bonne cause) fait que cette focalisation sur la réussite féminine ne fait le plus souvent que réactiver ces derniers. (Pourquoi une femme est-elle obligée d'accomplir des exploits au volant d'un véhicule et de toujours rivaliser avec un homme ? Tout cela pour trouver sa place à l'intérieur de l'univers de l'automobile.).

Autre recours guerrier, le travestissement ou le camouflage. Dans l'univers du travail, le personnage féminin, s'il n'est pas réduit à un simple faire valoir de l'homme, peut surgir sous les traits d'un mannequin célèbre ou bien de son clone, parangon de la femme-femme mais, caricature explicite du fait du décalage improbable entre l'éthos du personnage et la scène sociale qui le plus souvent le confronte à une assemblée improbable d'hommes soumis et serviles. Et, si parfois cette combattante cherche à inverser les rôles dans l'univers de la séduction (et donc ne pas en rester au statut d'objet) et qu'il lui arrive par malheur d'exprimer un désir et de passer à l'acte, elle devient une pécheresse (le confessionnal, 1000 de Jean Patou, 1988). Car en arrière plan, le masculin représente toujours le référent. Dans les univers équivalents, la mise en scène de l'homme se limite à l'évocation exclusive du territoire du produit; il travaille, conduit une voiture, séduit les femmes quasi "naturellement", sans manifester le moindre état d'âme (Egoïste Channel, 1990). À l'opposé, le discours concernant la femme doit se doter d'une étoffe narrative et représentionnelle plus ample. Cette dernière va systématiquement à la rencontre d'épreuves et d'obstacles étrangers à l'univers du produit, renvoyant le plus souvent l'héroïne à son identité socio-biologique de femme («Opel Tigra, la plus belle invention depuis la pilule», 2004). Dans ce jeu

consommateur, mais, en menant une OPA sur les programmes, le téléspectateur lui-même, sans doute avec plus de dommages collatéraux. 
d'affrontements et d'évitements, le recours aux procédés humoristiques ${ }^{11}$ apparaît tout à fait symptomatique du conflit de définition qui entoure l'identité sociale féminine dans ses représentations. Certains scénarios reposant sur la dérision voire la provocation relèvent parfois de cette idéologie régressive backlash, dénoncée par les féministes américaines, qui “jouerait" à remettre en question les avancées de la lutte des femmes («Babette, je la lie, je la fouette, et parfois elle passe à la casserole », 2000). Cet humour cynique très présent dans la publicité française repose sur un procédé délibéré d'ambiguïsation, une forme de convocation non-sérieuse ou bien de contournement du stéréotype qui de facto n'est jamais remis en question frontalement.

Dans ces espaces de non-droit féminin et de suprématie du masculin, la publicité télévisée construit ces personnages féminins de combattantes comme des membres d'une diaspora, figures immigrées, étrangères au territoire du produit et qui doivent laborieusement conquérir seules leur propre territoire.

Le troisième avatar est celui de l'icône. Il incarne d'un certain point de vue les finalités et les velléités prônées par un certain néo-féminisme qui, tout en revendiquant un territoire et une singularité spécifiquement féminine, en vient à naturaliser une "féminité" native.

À côté de ces compagnes soumises ou combattantes, cohabitent des figurations plus centripètes proposant des portraits de la femme-femme ou bien ceux de la femme-mère, constamment dépeintes comme porteuses d'une promesse d'épanouissement, de rapports équilibrés en rupture avec la mère marâtre ou la femme domestique, cantonnées au maintien de l'ordre ou à la gestion de l'espace domestique. Tout l'univers des soins du corps et de l'art cosmétique alimente cette image de soi de la femme. Cette figuration s'accompagne d'une dissolution de tout environnement social et simultanément d'une fétichisation du corps féminin, morcelé et découpé. Ce corps transfiguré y devient le seul attribut de la femme et son incarnation exclusive. Ce corps spéculaire $^{12}$, ce corps quasi balisé des femmes se présente comme la carte de visite obligée du genre («Sois belle et tais toi !»). Or, cet «être pour soi » que la publicité met en scène ne représente qu'un être de papier ou de discours car « ce corps de rêve », «cette peau et cette chevelure merveilleuses » sont des sortes de trophées somptuaires, réduits, comme le souligne Jean Baudrillard ${ }^{13}$, à leur pure valeur de signes. En effet, ces préceptes et recettes de parure, "essentiels" à l'éthos de la "vraie femme" sont autant de diktats quasi-autarciques qui sont rarement articulés à une visée pragmatique ou factitive. Ils apparaissent comme de purs rituels « de consommation de soi ${ }^{14}$ » à travers une panoplie du devoir être conforme totalement naturalisée. À aucun moment du reste, cette activité solitaire et réflexive n'implique une impulsion en direction d'un objet quelconque et l'esquisse de la silhouette d'un autre du désir, si ce n'est le reflet immémorial de Narcisse. Affublée de ce masque, l'identité féminine se confond avec

\footnotetext{
${ }^{11}$ Chabrol Claude Charaudeau Patrick (dir.), Questions de communication $n^{\circ} 10$, Humour et médias, définitions, genres et cultures, Nancy, Presses universitaires de Nancy, 2004.

${ }^{12}$ On peut observer toutefois que le corps féminin exposé dans la publicité télévisée (publicité à vocation familiale) est bien loin du corps érotisé des publicités de magazines ou de certaines affiches. C'est le plus souvent un corps aseptisé, un corps biologique dont on a évacué toute trace d'érotisme.

${ }^{13}$ Baudrillard Jean, Pour une critique de l'économie politique des signes, Paris, tel Gallimard, 1972.

${ }^{14}$ Quessada Dominique, La société de consommation de soi, Paris, Verticales, Le Seuil, 1999.
} 
une pure essence déréalisée, déniant et refoulant du même coup la condition empirique et plurielle de la femme réelle.

Au cour de cette reconfiguration du type idéal, une autre figure, celle de la femmemère trouve également sa place. Car entre-temps, l'enfant lui-même a subi une métamorphose, il n'est plus le garnement qui fuit et transgresse les commandements de la mère-marâtre, il est devenu le rejeton choyé du foyer épanoui dont il constitue désormais le centre. Image centripète de l'enfant roi et désiré d'une mère épanouie et non plus de cette femme, sujet mutique auquel s'adressait la voix rauque et virile de l'autorité, qui devait lui dicter, il y a peu encore, la bonne route et les conduites conformes.

\section{Conclusion}

Paradoxalement ces trois figures ne se rencontrent jamais. Sur le petit écran, comme sans doute dans l'expérience vécue de ses publics, l'identité féminine est structurellement fragmentée et chaque téléspectatrice doit jongler constamment avec plusieurs répertoires définitionnels divergents. Manifestement, la publicité à la télévision ne peut assurer un discours univoque et fédérateur, comme elle peut sans complexe l'assumer dans certains magazines féminins. Elle se doit d'y parler à tous et à toutes et s'évertuer à faire cohabiter ce choeur dissonant. En partie verrouillée par un droit d'entrée très élevé qui exclut les petites marques et peut-être les voies divergentes, la publicité de marque est victime également de la dérive généraliste du média télévisuel — de ce point de vue même les chaînes thématiques ont échoué dans leur stratégie de segmentation des audiences (à l'exception des chaînes pour enfants). En conséquence, la publicité télévisée doit cultiver le consensus.

Cet encadrement des programmes qu'elle assure remplit une fonction purement ornementale qui, sans écart et sans provocation, opère docilement la reconduction des normes dominantes. Aussi, les mondes possibles qu'elle nous fabule sont loin d'être avant-gardistes ou transgressifs, ils sont surtout éminemment réalistes, fruits d'un compromis entre les visées et les pratiques des producteurs et les "grammaires de reconnaissance ${ }^{15}$ " des publics. Mais ces fabulations sont aussi des marques d'appartenance qui dessinent les contours de ces «communautés imaginées ${ }^{16}$ » qui hantent la sphère publique de nos sociétés démocratiques. Car derrière la libre circulation des marchandises et la mise en concurrence des marques, ce sont aussi les fictions et les trajectoires conflictuelles de nos identités sociales qui s'écrivent.

\footnotetext{
${ }^{15}$ Veron, Eliséo, "Presse écrite et théorie des discours sociaux : production, réception, régulation " in Charaudeau, Patrick (dir.) La Presse, Produit, Production, Réception, Paris, Didier-Érudition, 1988.

${ }^{16}$ Anderson Benedict, L'imaginaire national. Réflexions sur l'origine et l'essor du nationalisme, Paris ; La Découverte/poche, 2002.
} 